\title{
Coupled coincidence point and common coupled fixed point results in cone $b$-metric spaces
}

\section{Zaid Mohammed Fadail ${ }^{*}$ and Abd Ghafur Bin Ahmad}

\section{"Correspondence:}

zaid_fatail@yahoo.com

School of Mathematical Sciences,

Faculty of Science and Technology,

Universiti Kebangsaan Malaysia,

Bangi, Selangor Darul Ehsan 43600,

Malaysia

\begin{abstract}
In this paper, we establish common coupled fixed point and coupled fixed point theorems in cone $b$-metric spaces. The presented theorems extend and generalize several well-known comparable results in literature. We supply some examples to elucidate our obtained results.
\end{abstract}

Keywords: cone $b$-metric spaces; coupled fixed points; coupled coincidence points; common coupled fixed points

\section{Introduction and preliminaries}

In [1], Bakhtin introduced $b$-metric spaces (or metric-type spaces) as a generalization of metric spaces. He evidenced the contraction mapping principle in $b$-metric spaces that generalized the famous Banach contraction principle in metric spaces. From that time on, manifold papers have treated fixed point theory or the variational principle for single-valued and multi-valued operators in $b$-metric spaces (see [2-7] and the references therein).

Ordered normed spaces, cones and topical functions have applications in applied mathematics, for instance, in using Newton's approximation method [8-12] and optimization theory [13, 14]. In the mid-twentieth century [9], $k$-metric and $k$-normed spaces were established (see also $[11,12]$ ) by replacing an ordered Banach space instead of the set of real numbers, as the codomain for a metric. Due to defining convergent and Cauchy sequences in terms of interior points of the underlying cone, Huang and Zhang [15] re-introduced such spaces under the name of cone metric space. Even though they used only normal cones, nonnormal cones can be used as well in such a way but by taking into the consideration that the Sandwich theorem and continuity of the metric may not hold. Some fixed point theorems for contractive-type mappings in cone metric spaces have been substantiated; for more details, see [16-25].

As a generalization of $b$-metric spaces and cone metric spaces, Hussain and Shah [26] announced cone $b$-metric spaces, which was in 2011. They built up some topological properties in such spaces and upgraded some latest results about KKM mappings in the setting of a cone $b$-metric space. Hussain and Shah [26] have done initial work that stimulated many authors to prove fixed point theorems, as well as common fixed point theorems for two or more mappings on cone $b$-metric spaces (see [27-30] and the references therein). 
The following will be needed in the sequel.

Let $E$ be a real Banach space and let $\theta$ denote the zero element in $E$. A cone $P$ is a subset of $E$ such that:

1. $P$ is nonempty set closed and $P \neq\{\theta\}$.

2. If $a, b$ are nonnegative real numbers and $x, y \in P$, then $a x+b y \in P$.

3. $x \in P$ and $-x \in P$ implies $x=\theta$.

For any cone $P \subset E$, the partial ordering $\preceq$ with respect to $P$ is defined by $x \preceq y$ if and only if $y-x \in P$. The notation of $\prec$ stands for $x \preceq y$ but $x \neq y$. Also, we used $x \ll y$ to indicate that $y-x \in \operatorname{int} P$, where int $P$ denotes the interior of $P$. A cone $P$ is called normal if there exists a number $K$ such that

$$
\theta \preceq x \preceq y \quad \Longrightarrow \quad\|x\| \leq K\|y\|
$$

for all $x, y \in E$. The least positive number $K$ satisfying the above condition is called the normal constant of $P$. Throughout this paper, we do not impose the normality condition for the cones, but the only assumption is that the cone $P$ is solid, that is, int $P \neq \emptyset$.

Definition 1.1 [26] Let $X$ be a nonempty set and $E$ be a real Banach space equipped with the partial ordering $\preceq$ with respect to the cone $P$. A vector-valued function $d: X \times X \rightarrow$ $E$ is said to be a cone $b$-metric function on $X$ with the constant $s \geq 1$ if the following conditions are satisfied:

1. $\theta \preceq d(x, y)$ for all $x, y \in X$ and $d(x, y)=\theta$ if and only if $x=y$,

2. $d(x, y)=d(y, x)$ for all $x, y \in X$,

3. $d(x, y) \preceq s(d(x, y)+d(y, z))$ for all $x, y, z \in X$.

Then the pair $(X, d)$ is called a cone $b$-metric space (or a cone metric-type space); we will use the first mentioned term.

Observe that if $s=1$ then the ordinary triangle inequality in a cone metric space is satisfied; however, it does not hold true when $s>1$. Thus the class of tvs-cone $b$-metric spaces is effectively larger than that of the ordinary cone metric spaces. That is, every cone metric space is a cone $b$-metric space, but the converse need not be true. The following examples illustrate the above remarks.

Example 1.2 [29] Let $X=\{-1,0,1\}, E=\mathbb{R}^{2}, P=\{(x, y): x \geq 0, y \geq 0\}$. Define $d: X \times X \rightarrow P$ by $d(x, y)=d(y, x)$ for all $x, y \in X, d(x, x)=\theta, x \in X$ and $d(-1,0)=(3,3), d(-1,1)=d(0,1)=$ $(1,1)$. Then $(X, d)$ is a complete cone $b$-metric space, but the triangle inequality is not satisfied. Indeed, we have that $d(-1,1)+d(1,0)=(1,1)+(1,1)=(2,2) \prec(3,3)=d(-1,0)$. It is not hard to verify that $s=\frac{3}{2}$.

The following example is a modification of Example 3 from [31].

Example 1.3 Let $X=\mathbb{N} \cup\{\infty\}, E=\mathbb{R}^{2}$ and $P=\{(x, y) \in E: x \geq 0, y \geq 0\}$. Define $d: X \times$ $X \rightarrow E$ by

$$
d(x, y)= \begin{cases}(0,0) & \text { if } x=y, \\ \left(\left|\frac{1}{x}-\frac{1}{y}\right|,\left|\frac{1}{x}-\frac{1}{y}\right|\right) & \text { if } x \text { and } y \text { are even or } x y=\infty, \\ (5,5) & \text { if } x \text { and } y \text { are odd and } x \neq y, \\ (2,2) & \text { otherwise. }\end{cases}
$$


Then $(X, d)$ is a cone $b$-metric space with the coefficient $s=3$. But it is not a cone metric space since the triangle inequality is not satisfied. Indeed, $(5,5)=d(1,3) \succ d(1,2)+d(2,3)=$ $(2,2)+(2,2)=(4,4)$.

Definition 1.4 [26] Let $(X, d)$ be a cone $b$-metric space, and let $\left\{x_{n}\right\}$ be a sequence in $X$ and $x \in X$.

1. For all $c \in E$ with $\theta \ll c$, if there exists a positive integer $N$ such that $d\left(x_{n}, x\right) \ll c$ for all $n>N$, then $x_{n}$ is said to be convergent and $x$ is the limit of $\left\{x_{n}\right\}$. We denote this by $x_{n} \rightarrow x$.

2. For all $c \in E$ with $\theta \ll c$, if there exists a positive integer $N$ such that $d\left(x_{n}, x_{m}\right) \ll c$ for all $n, m>N$, then $\left\{x_{n}\right\}$ is called a Cauchy sequence in $X$.

3. A cone metric space $(X, d)$ is called complete if every Cauchy sequence in $X$ is convergent.

The following lemma is helpful in proving our results.

\section{Lemma 1.5 [24]}

1. If $E$ is a real Banach space with $a$ cone $P$ and $a \preceq \lambda a$, where $a \in P$ and $0 \leq \lambda<1$, then $a=\theta$.

2. If $c \in \operatorname{int} P, \theta \preceq a_{n}$ and $a_{n} \rightarrow \theta$, then there exists a positive integer $N$ such that $a_{n} \ll c$ for all $n \geq N$.

3. If $a \preceq b$ and $b \ll c$, then $a \ll c$.

4. If $\theta \preceq u \ll c$ for each $\theta \ll c$, then $u=\theta$.

Recall the following definitions.

Definition 1.6 [32] An element $(x, y) \in X^{2}$ is said to be a coupled fixed point of the mapping $F: X^{2} \rightarrow X$ if $F(x, y)=x$ and $F(y, x)=y$.

Definition 1.7 [33] An element $(x, y) \in X^{2}$ is called

1. a coupled coincidence point of mappings $F: X^{2} \rightarrow X$ and $g: X \rightarrow X$ if $g x=F(x, y)$ and $g y=F(y, x)$, and $(g x, g y)$ is called a coupled point of coincidence;

2. a common coupled fixed point of mappings $F: X^{2} \rightarrow X$ and $g: X \rightarrow X$ if $x=g x=F(x, y)$ and $y=g y=F(y, x)$.

Definition 1.8 [25] The mappings $F: X^{2} \rightarrow X$ and $g: X \rightarrow X$ are called $w$-compatible if $g(F(x, y))=F(g x, g y)$ whenever $g x=F(x, y)$ and $g y=F(y, x)$.

\section{Coupled coincidence point results}

In this section, we prove some coupled coincidence point results in cone $b$-metric spaces.

Theorem 2.1 Let $(X, d)$ be a cone b-metric space with the coefficient $s \geq 1$ relative to a solid cone P. Let $F: X^{2} \rightarrow X$ and $g: X \rightarrow X$ be two mappings and suppose that there exist nonnegative constants $a_{i} \in[0,1), i=1,2, \ldots, 10$, with $(s+1)\left(a_{1}+a_{2}+a_{3}+a_{4}\right)+s(s+1)\left(a_{5}+\right.$ $\left.a_{6}+a_{7}+a_{8}\right)+2 s\left(a_{9}+a_{10}\right)<2$ and $\sum_{i=1}^{10} a_{i}<1$ such that the following contractive condition 
holds for all $x, y, u, v \in X$ :

$$
\begin{aligned}
d(F(x, y), F(u, v)) \leq & {\left[a_{1} d(g x, F(x, y))+a_{2} d(g y, F(y, x))\right] } \\
& +\left[a_{3} d(g u, F(u, v))+a_{4} d(g v, F(v, u))\right] \\
& +\left[a_{5} d(g x, F(u, v))+a_{6} d(g y, F(v, u))\right] \\
& +\left[a_{7} d(g u, F(x, y))+a_{8} d(g v, F(y, x))\right] \\
& +\left[a_{9} d(g x, g u)+a_{10} d(g y, g v)\right] .
\end{aligned}
$$

If $F\left(X^{2}\right) \subseteq g(X)$ and $g(X)$ is a complete subspace of $X$, then $F$ and $g$ have a coupled coincidence point $\left(x^{*}, y^{*}\right) \in X^{2}$.

Proof Choose $x_{0}, y_{0} \in X$. Set $g x_{1}=F\left(x_{0}, y_{0}\right), g y_{1}=F\left(y_{0}, x_{0}\right)$, this can be done because $F\left(X^{2}\right) \subseteq g(X)$. Continuing this process, we obtain two sequences $\left\{x_{n}\right\},\left\{y_{n}\right\}$ such that $g x_{n+1}=F\left(x_{n}, y_{n}\right)$ and $g y_{n+1}=F\left(y_{n}, x_{n}\right)$. Then we have

$$
\begin{aligned}
d\left(g x_{n}, g x_{n+1}\right)= & d\left(F\left(x_{n-1}, y_{n-1}\right), F\left(x_{n}, y_{n}\right)\right) \\
\preceq & {\left[a_{1} d\left(g x_{n-1}, F\left(x_{n-1}, y_{n-1}\right)\right)+a_{2} d\left(g y_{n-1}, F\left(y_{n-1}, x_{n-1}\right)\right)\right] } \\
& +\left[a_{3} d\left(g x_{n}, F\left(x_{n}, y_{n}\right)\right)+a_{4} d\left(g y_{n}, F\left(y_{n}, x_{n}\right)\right)\right] \\
& +\left[a_{5} d\left(g x_{n-1}, F\left(x_{n}, y_{n}\right)\right)+a_{6} d\left(g y_{n-1}, F\left(y_{n}, x_{n}\right)\right)\right] \\
& +\left[a_{7} d\left(g x_{n}, F\left(x_{n-1}, y_{n-1}\right)\right)+a_{8} d\left(g y_{n}, F\left(y_{n-1}, x_{n-1}\right)\right)\right] \\
& +\left[a_{9} d\left(g x_{n-1}, g x_{n}\right)+a_{10} d\left(g y_{n-1}, g y_{n}\right)\right] .
\end{aligned}
$$

So that

$$
\begin{aligned}
d\left(g x_{n}, g x_{n+1}\right)= & d\left(F\left(x_{n-1}, y_{n-1}\right), F\left(x_{n}, y_{n}\right)\right) \\
\subseteq & {\left[a_{1} d\left(g x_{n-1}, g x_{n}\right)+a_{2} d\left(g y_{n-1}, g y_{n}\right)\right]+\left[a_{3} d\left(g x_{n}, g x_{n+1}\right)+a_{4} d\left(g y_{n}, g y_{n+1}\right)\right] } \\
& +\left[a_{5} d\left(g x_{n-1}, g x_{n+1}\right)+a_{6} d\left(g y_{n-1}, g y_{n+1}\right)\right] \\
& +\left[a_{7} d\left(g x_{n}, g x_{n}\right)+a_{8} d\left(g y_{n}, g y_{n}\right)\right] \\
& +\left[a_{9} d\left(g x_{n-1}, g x_{n}\right)+a_{10} d\left(g y_{n-1}, g y_{n}\right)\right] \\
\subseteq & {\left[a_{1} d\left(g x_{n-1}, g x_{n}\right)+a_{2} d\left(g y_{n-1}, g y_{n}\right)\right]+\left[a_{3} d\left(g x_{n}, g x_{n+1}\right)+a_{4} d\left(g y_{n}, g y_{n+1}\right)\right] } \\
& +\left[s a_{5}\left(d\left(g x_{n-1}, g x_{n}\right)+d\left(g x_{n}, g x_{n+1}\right)\right)\right. \\
& \left.+s a_{6}\left(d\left(g y_{n-1}, g y_{n}\right)+d\left(g y_{n}, g y_{n+1}\right)\right)\right] \\
& +\left[a_{9} d\left(g x_{n-1}, g x_{n}\right)+a_{10} d\left(g y_{n-1}, g y_{n}\right)\right] .
\end{aligned}
$$

Hence,

$$
\begin{aligned}
d\left(g x_{n}, g x_{n+1}\right) \leq & {\left[\left(a_{1}+a_{5} s+a_{9}\right) d\left(g x_{n-1}, g x_{n}\right)+\left(a_{2}+a_{6} s+a_{10}\right) d\left(g y_{n-1}, g y_{n}\right)\right] } \\
& +\left[\left(a_{3}+a_{5} s\right) d\left(g x_{n}, g x_{n+1}\right)+\left(a_{4}+a_{6} s\right) d\left(g y_{n}, g y_{n+1}\right)\right] .
\end{aligned}
$$


Similarly, we can prove that

$$
\begin{aligned}
d\left(g y_{n}, g y_{n+1}\right) \preceq & {\left[\left(a_{1}+s a_{5}+a_{9}\right) d\left(g y_{n-1}, g y_{n}\right)+\left(a_{2}+s a_{6}+a_{10}\right) d\left(g x_{n-1}, g x_{n}\right)\right] } \\
& +\left[\left(a_{3}+s a_{5}\right) d\left(g y_{n}, g y_{n+1}\right)+\left(a_{4}+s a_{6}\right) d\left(g x_{n}, g x_{n+1}\right)\right] .
\end{aligned}
$$

Put

$$
d_{n}=d\left(g x_{n}, g x_{n+1}\right)+d\left(g y_{n}, g y_{n+1}\right) .
$$

Adding inequalities (2.1) and (2.2), one can assert that

$$
d_{n} \preceq\left(a_{1}+a_{2}+s a_{5}+s a_{6}+a_{9}+a_{10}\right) d_{n-1}+\left(a_{3}+a_{4}+s a_{5}+s a_{6}\right) d_{n} .
$$

On the other hand, we have

$$
\begin{aligned}
d\left(g x_{n+1}, g x_{n}\right)= & d\left(F\left(x_{n}, y_{n}\right), F\left(x_{n-1}, y_{n-1}\right)\right) \\
\subseteq & {\left[a_{1} d\left(g x_{n}, F\left(x_{n}, y_{n}\right)\right)+a_{2} d\left(g y_{n}, F\left(y_{n}, x_{n}\right)\right)\right] } \\
& +\left[a_{3} d\left(g x_{n-1}, F\left(x_{n-1}, y_{n-1}\right)\right)+a_{4} d\left(g y_{n-1}, F\left(y_{n-1}, x_{n-1}\right)\right)\right] \\
& +\left[a_{5} d\left(g x_{n}, F\left(x_{n-1}, y_{n-1}\right)\right)+a_{6} d\left(g y_{n}, F\left(y_{n-1}, x_{n-1}\right)\right)\right] \\
& +\left[a_{7} d\left(g x_{n-1}, F\left(x_{n}, y_{n}\right)\right)+a_{8} d\left(g y_{n-1}, F\left(y_{n}, x_{n}\right)\right)\right] \\
& +\left[a_{9} d\left(g x_{n}, g x_{n-1}\right)+a_{10} d\left(g y_{n}, g y_{n-1}\right)\right] .
\end{aligned}
$$

So that

$$
\begin{aligned}
d\left(g x_{n+1}, g x_{n}\right)= & d\left(F\left(x_{n}, y_{n}\right), F\left(x_{n-1}, y_{n-1}\right)\right)+\left[a_{1} d\left(g x_{n}, g x_{n+1}\right)+a_{2} d\left(g y_{n}, g y_{n+1}\right)\right] \\
\leq & \left.a_{3} d\left(g x_{n-1}, g x_{n}\right)+a_{4} d\left(g y_{n-1}, g y_{n}\right)\right] \\
& +\left[a_{5} d\left(g x_{n}, g x_{n}\right)+a_{6} d\left(g y_{n}, g y_{n}\right)\right] \\
& +\left[a_{7} d\left(g x_{n-1}, g x_{n+1}\right)+a_{8} d\left(g y_{n-1}, g y_{n+1}\right)\right] \\
& +\left[a_{9} d\left(g x_{n}, g x_{n-1}\right)+a_{10} d\left(g y_{n}, g y_{n-1}\right)\right] \\
\subseteq & {\left[a_{1} d\left(g x_{n}, g x_{n+1}\right)+a_{2} d\left(g y_{n}, g y_{n+1}\right)\right] } \\
& +\left[a_{3} d\left(g x_{n-1}, g x_{n}\right)+a_{4} d\left(g y_{n-1}, g y_{n}\right)\right] \\
& +\left[s a_{7}\left(d\left(g x_{n-1}, g x_{n}\right)+d\left(g x_{n}, g x_{n+1}\right)\right)\right. \\
& \left.+s a_{8}\left(d\left(g y_{n-1}, g y_{n}\right)+d\left(g y_{n}, g y_{n+1}\right)\right)\right] \\
& +\left[a_{9} d\left(g x_{n-1}, g x_{n}\right)+a_{10} d\left(g y_{n-1}, g y_{n}\right)\right] .
\end{aligned}
$$

Hence,

$$
\begin{aligned}
d\left(g x_{n+1}, g x_{n}\right) \leq & {\left[\left(a_{3}+s a_{7}+a_{9}\right) d\left(g x_{n-1}, g x_{n}\right)+\left(a_{4}+s a_{8}+a_{10}\right) d\left(g y_{n-1}, g y_{n}\right)\right] } \\
& +\left[\left(a_{1}+s a_{7}\right) d\left(g x_{n}, g x_{n+1}\right)+\left(a_{2}+s a_{8}\right) d\left(g y_{n}, g y_{n+1}\right)\right] .
\end{aligned}
$$


Similarly,

$$
\begin{aligned}
d\left(g y_{n+1}, g y_{n}\right) \preceq & {\left[\left(a_{3}+s a_{7}+a_{9}\right) d\left(g y_{n-1}, g y_{n}\right)+\left(a_{4}+s a_{8}+a_{10}\right) d\left(g x_{n-1}, g x_{n}\right)\right] } \\
& +\left[\left(a_{1}+s a_{7}\right) d\left(g y_{n}, g y_{n+1}\right)+\left(a_{2}+s a_{8}\right) d\left(g x_{n}, g x_{n+1}\right)\right] .
\end{aligned}
$$

Adding inequalities (2.4) and (2.5), one can assert that

$$
d_{n} \preceq\left(a_{3}+a_{4}+s a_{7}+s a_{8}+a_{9}+a_{10}\right) d_{n-1}+\left(a_{1}+a_{2}+s a_{7}+s a_{8}\right) d_{n} .
$$

Finally, from (2.3) and (2.6), we have

$$
\begin{aligned}
2 d_{n} \preceq & \left(a_{1}+a_{2}+a_{3}+a_{4}+s a_{5}+s a_{6}+s a_{7}+s a_{8}+2\left(a_{9}+a_{10}\right)\right) d_{n-1} \\
& +\left(a_{1}+a_{2}+a_{3}+a_{4}+s a_{5}+s a_{6}+s a_{7}+s a_{8}\right) d_{n},
\end{aligned}
$$

that is,

$$
d_{n} \preceq h d_{n-1},
$$

where $h=\frac{\left(a_{1}+a_{2}+a_{3}+a_{4}+s a_{5}+s a_{6}+s a_{7}+s a_{8}+2\left(a_{9}+a_{10}\right)\right)}{2-\left(a_{1}+a_{2}+a_{3}+a_{4}+s a_{5}+s a_{6}+s a_{7}+s a_{8}\right)}<\frac{1}{s}$.

Consequently, we have

$$
\begin{aligned}
d_{n} & \leq h d_{n-1} \\
& \leq h^{2} d_{n-2} \\
& \preceq h^{3} d_{n-3} \\
& \vdots \\
& \preceq h^{n} d_{0} .
\end{aligned}
$$

Let $m>n \geq 1$. It follows that

$$
d\left(g x_{n}, g x_{m}\right) \preceq s d\left(g x_{n}, g x_{n+1}\right)+s^{2} d\left(g x_{n+1}, g x_{n+2}\right)+\cdots+s^{m-n} d\left(g x_{m-1}, g x_{m}\right)
$$

and

$$
d\left(g y_{n}, g y_{m}\right) \preceq s d\left(g y_{n}, g y_{n+1}\right)+s^{2} d\left(g y_{n+1}, g x_{n+2}\right)+\cdots+s^{m-n} d\left(g y_{m-1}, g y_{m}\right) .
$$

Now, (2.7) and $s h<1$ imply that

$$
\begin{aligned}
d\left(g x_{n}, g x_{m}\right)+d\left(g y_{n}, g y_{m}\right) & \preceq s d_{n}+s^{2} d_{n+1}+\cdots+s^{m-n} d_{m-1} \\
& \preceq s h^{n} d_{0}+s^{2} h^{n+1} d_{0}+\cdots+s^{m-n} h^{m-1} d_{0} \\
& =\left(s h^{n}+s^{2} h^{n+1}+\cdots+s^{m-n} h^{m-1}\right) d_{0} \\
& =s h^{n}\left(1+s h+(s h)^{2}+\cdots+(s h)^{m-n-1}\right) d_{0} \\
& \preceq \frac{s h^{n}}{1-s h} d_{0} \rightarrow \theta \quad \text { as } n \rightarrow \infty .
\end{aligned}
$$


According to Lemma 1.5(2), and for any $c \in E$ with $c \gg \theta$, there exists $N_{0} \in \mathbb{N}$ such that for any $n>N_{0}, \frac{h^{n}}{1-h} d_{0} \ll c$. Furthermore, from (2.8) and for any $m>n>N_{0}$, Lemma 1.5(3) shows that

$$
d\left(g x_{n}, g x_{m}\right)+d\left(g y_{n}, g y_{m}\right) \ll c,
$$

which implies that

$$
d\left(g x_{n}, g x_{m}\right) \ll c
$$

and

$$
d\left(g y_{n}, g y_{m}\right) \ll c .
$$

Hence, by Definition 1.4(2), $\left\{g x_{n}\right\}$ and $\left\{g y_{n}\right\}$ are Cauchy sequences in $g(X)$. Since $g(X)$ is complete, there exist $x^{*}$ and $y^{*} \in X$ such that $g x_{n} \rightarrow g x^{*}$ and $g y_{n} \rightarrow g y^{*}$ as $n \rightarrow \infty$.

On the other hand,

$$
\begin{aligned}
d\left(F\left(x^{*}, y^{*}\right), g x^{*}\right) \subseteq & s\left(d\left(F\left(x^{*}, y^{*}\right), g x_{n+1}\right)+d\left(g x_{n+1}, g x^{*}\right)\right) \\
= & s\left(d\left(F\left(x^{*}, y^{*}\right), F\left(x_{n}, y_{n}\right)\right)+d\left(g x_{n+1}, g x^{*}\right)\right) \\
\leq & s\left[a_{1} d\left(g x^{*}, F\left(x^{*}, y^{*}\right)\right)+a_{2} d\left(g y^{*}, F\left(y^{*}, x^{*}\right)\right)\right] \\
& +s\left[a_{3} d\left(g x_{n}, F\left(x_{n}, y_{n}\right)\right)+a_{4} d\left(g y_{n}, F\left(y_{n}, x_{n}\right)\right)\right] \\
& +s\left[a_{5} d\left(g x^{*}, F\left(x_{n}, y_{n}\right)\right)+a_{6} d\left(g y^{*}, F\left(y_{n}, x_{n}\right)\right)\right] \\
& +s\left[a_{7} d\left(g x_{n}, F\left(x^{*}, y^{*}\right)\right)+a_{8} d\left(g y_{n}, F\left(y^{*}, x^{*}\right)\right)\right] \\
& +s\left[a_{9} d\left(g x^{*}, g x_{n}\right)+a_{10} d\left(g y^{*}, g y_{n}\right)\right]+s d\left(g x_{n+1}, g x^{*}\right) \\
\leq & s\left[a_{1} d\left(F\left(x^{*}, y^{*}\right), g x^{*}\right)+a_{2} d\left(F\left(y^{*}, x^{*}\right), g y^{*}\right)\right] \\
& +s\left[s a_{3} d\left(g x_{n}, g x^{*}\right)+s a_{3} d\left(g x^{*}, g x_{n+1}\right)\right. \\
& \left.+s a_{4} d\left(g y_{n}, g y^{*}\right)+s a_{4} d\left(g y^{*}, g y_{n+1}\right)\right] \\
& +s\left[a_{5} d\left(g x^{*}, g x_{n+1}\right)+a_{6} d\left(g y^{*}, g y_{n+1}\right)\right] \\
& +s\left[s a_{7} d\left(g x_{n}, g x^{*}\right)+s a_{7} d\left(g x^{*}, F\left(x^{*}, y^{*}\right)\right)\right. \\
& \left.+s a_{8} d\left(g y_{n}, g y^{*}\right)+s a_{8} d\left(g y^{*}, F\left(y^{*}, x^{*}\right)\right)\right] \\
& +s\left[a_{9} d\left(g x^{*}, g x_{n}\right)+a_{10} d\left(g y^{*}, g y_{n}\right)\right]+s d\left(g x_{n+1}, g x^{*}\right) \\
= & s\left[a_{1} d\left(F\left(x^{*}, y^{*}\right), g x^{*}\right)+a_{2} d\left(F\left(y^{*}, x^{*}\right), g y^{*}\right)\right] \\
& +s\left[s a_{3} d\left(g x_{n}, g x^{*}\right)+s a_{3} d\left(g x_{n+1}, g x^{*}\right)\right. \\
& \left.+s a_{4} d\left(g y_{n}, g y^{*}\right)+s a_{4} d\left(g y_{n+1}, g y^{*}\right)\right] \\
& +s\left[a_{5} d\left(g x_{n+1}, g x^{*}\right)+a_{6} d\left(g y_{n+1}, g y^{*}\right)\right] \\
& +s\left[s a_{7} d\left(g x_{n}, g x^{*}\right)+s a_{7} d\left(F\left(x^{*}, y^{*}\right), g x^{*}\right)\right. \\
& \left.+s a_{8} d\left(g y_{n}, g y^{*}\right)+s a_{8} d\left(F\left(y^{*}, x^{*}\right), g y^{*}\right)\right] \\
& +s\left[a_{9} d\left(g x_{n}, g x^{*}\right)+a_{10} d\left(g y_{n}, g y^{*}\right)\right]+s d\left(g x_{n+1}, g x^{*}\right) . \\
&
\end{aligned}
$$


Hence,

$$
\begin{aligned}
d\left(F\left(x^{*}, y^{*}\right), g x^{*}\right) \preceq & \left(s a_{1}+s^{2} a_{7}\right) d\left(F\left(x^{*}, y^{*}\right), g x^{*}\right)+\left(a_{2}+s^{2} a_{8}\right) d\left(F\left(y^{*}, x^{*}\right), g y^{*}\right) \\
& +\left(s^{2} a_{3}+s^{2} a_{7}+s a_{9}\right) d\left(g x_{n}, g x^{*}\right)+\left(s^{2} a_{3}+s a_{5}+s\right) d\left(g x_{n+1}, g x^{*}\right) \\
& +\left(s^{2} a_{4}+s^{2} a_{8}+s a_{10}\right) d\left(g y_{n}, g y^{*}\right)+\left(s^{2} a_{4}+s a_{6}\right) d\left(g y_{n+1}, g y^{*}\right) .
\end{aligned}
$$

Similarly,

$$
\begin{aligned}
d\left(F\left(y^{*}, x^{*}\right), g y^{*}\right) \subseteq & \left(s a_{1}+s^{2} a_{7}\right) d\left(F\left(y^{*}, x^{*}\right), g y^{*}\right)+\left(a_{2}+s^{2} a_{8}\right) d\left(F\left(x^{*}, y^{*}\right), g x^{*}\right) \\
& +\left(s^{2} a_{3}+s^{2} a_{7}+s a_{9}\right) d\left(g y_{n}, g y^{*}\right)+\left(s^{2} a_{3}+s a_{5}+s\right) d\left(g y_{n+1}, g y^{*}\right) \\
& +\left(s^{2} a_{4}+s^{2} a_{8}+s a_{10}\right) d\left(g x_{n}, g x^{*}\right)+\left(s^{2} a_{4}+s a_{6}\right) d\left(g x_{n+1}, g x^{*}\right) .
\end{aligned}
$$

Put

$$
\delta=d\left(F\left(x^{*}, y^{*}\right), g x^{*}\right)+d\left(F\left(y^{*}, x^{*}\right), g y^{*}\right) .
$$

Adding the above inequalities, we get

$$
\begin{aligned}
\delta \preceq & \left(s a_{1}+s^{2} a_{7}+a_{2}+s^{2} a_{8}\right) \delta \\
& +\left(s^{2} a_{3}+s^{2} a_{7}+s a_{9}+s^{2} a_{4}+s^{2} a_{8}+s a_{10}\right) d\left(g x_{n}, g x^{*}\right) \\
& +\left(s^{2} a_{3}+s^{2} a_{7}+s a_{9}+s^{2} a_{4}+s^{2} a_{8}+s a_{10}\right) d\left(g y_{n}, g y^{*}\right) \\
& +\left(s^{2} a_{3}+s a_{5}+s+s^{2} a_{4}+s a_{6}\right) d\left(g x_{n+1}, g x^{*}\right) \\
& +\left(s^{2} a_{3}+s a_{5}+s+s^{2} a_{4}+s a_{6}\right) d\left(g y_{n+1}, g y^{*}\right) .
\end{aligned}
$$

Then

$$
\delta \preceq \frac{B}{1-A} d\left(g x_{n}, g x^{*}\right)+\frac{B}{1-A} d\left(g y_{n}, g y^{*}\right)+\frac{C}{1-A} d\left(g x_{n+1}, g x^{*}\right)+\frac{C}{1-A} d\left(g y_{n+1}, g y^{*}\right),
$$

where $A=s a_{1}+s^{2} a_{7}+a_{2}+s^{2} a_{8}, B=s^{2} a_{3}+s^{2} a_{7}+s a_{9}+s^{2} a_{4}+s^{2} a_{8}+s a_{10}$ and $C=s^{2} a_{3}+s a_{5}+$ $s+s^{2} a_{4}+s a_{6}$. Since $g x_{n} \rightarrow g x^{*}$ and $g y_{n} \rightarrow g y^{*}$ as $n \rightarrow \infty$, then by Definition 1.4(1) and for $c \gg \theta$, there exists $N_{0} \in \mathbb{N}$ such that for all $n>N_{0}, d\left(g x_{n}, g x^{*}\right) \ll c \frac{1-A}{4 B}, d\left(g y_{n}, g y^{*}\right) \ll c \frac{1-A}{4 B}$, $d\left(g x_{n+1}, g x^{*}\right) \ll c \frac{1-A}{4 C}$ and $d\left(g y_{n+1}, g y^{*}\right) \ll c \frac{1-A}{4 C}$. Hence,

$$
\begin{aligned}
\delta & \preceq \frac{B}{A} d\left(g x_{n}, g x^{*}\right)+\frac{B}{A} d\left(g y_{n}, g y^{*}\right)+\frac{B}{C} d\left(g x_{n+1}, g x^{*}\right)+\frac{B}{C} d\left(g y_{n+1}, g y^{*}\right) \\
& \ll c \frac{1-A}{4 B} \frac{B}{1-A}+c \frac{1-A}{4 B} \frac{B}{1-A}+c \frac{1-A}{4 C} \frac{C}{1-A}+c \frac{1-A}{4 C} \frac{C}{1-A} \\
& =\frac{c}{4}+\frac{c}{4}+\frac{c}{4}+\frac{c}{4} \\
& =c .
\end{aligned}
$$

Now, according to Lemma 1.5(4), it follows that $\delta=\theta$, that is, $\left(F\left(x^{*}, y^{*}\right), g x^{*}\right)+d\left(F\left(y^{*}, x^{*}\right)\right.$, $\left.g y^{*}\right)=\theta$, which implies that $\left(F\left(x^{*}, y^{*}\right), g x^{*}\right)=\theta$ and $d\left(F\left(y^{*}, x^{*}\right), g y^{*}\right)=\theta$. Hence, $g x^{*}=$ $F\left(x^{*}, y^{*}\right)$ and $g y^{*}=F\left(y^{*}, x^{*}\right)$. Therefore $\left(x^{*}, y^{*}\right)$ is a coupled coincidence point of $F$ and $g$. 
Remark 2.2 Theorem 2.1 extends and generalizes Theorem 2.4 of Abbas et al. [25] to cone $b$-metric spaces.

Corollary 2.3 Let $(X, d)$ be a cone $b$-metric space with the coefficient $s \geq 1$ relative to a solid cone $P$. Let $F: X^{2} \rightarrow X$ and $g: X \rightarrow X$ be two mappings and suppose that there exist nonnegative constants $k, l$ with $k+l<\frac{1}{s}$ such that the following contractive condition holds for all $x, y, u, v \in X$ :

$$
d(F(x, y), F(u, v)) \preceq k d(g x, g u)+l d(g y, g v) .
$$

If $F\left(X^{2}\right) \subseteq g(X)$ and $g(X)$ is a complete subspace of $X$, then $F$ and $g$ have a coupled coincidence point $\left(x^{*}, y^{*}\right) \in X^{2}$.

Corollary 2.4 Let $(X, d)$ be a cone $b$-metric space with the coefficient $s \geq 1$ relative to a solid cone P. Let $F: X^{2} \rightarrow X$ and $g: X \rightarrow X$ be two mappings and suppose that there exist nonnegative constants $k, l$ with $k+l<\frac{2}{s+1}$ such that the following contractive condition holds for all $x, y, u, v \in X$ :

$$
d(F(x, y), F(u, v)) \preceq k d(g x, F(x, y))+l d(g u, F(u, v)) .
$$

If $F\left(X^{2}\right) \subseteq g(X)$ and $g(X)$ is a complete subspace of $X$, then $F$ and $g$ have a coupled coincidence point $\left(x^{*}, y^{*}\right) \in X^{2}$.

Corollary 2.5 Let $(X, d)$ be a cone b-metric space with the coefficient $s \geq 1$ relative to a solid cone P. Let $F: X^{2} \rightarrow X$ and $g: X \rightarrow X$ be two mappings and suppose that there exist nonnegative constants $k, l$ with $k+l<\frac{2}{s(s+1)}$ such that the following contractive condition holds for all $x, y, u, v \in X$ :

$$
d(F(x, y), F(u, v)) \preceq k d(g x, F(u, v))+l d(g u, F(x, y)) .
$$

If $F\left(X^{2}\right) \subseteq g(X)$ and $g(X)$ is a complete subspace of $X$, then $F$ and $g$ have a coupled coincidence point $\left(x^{*}, y^{*}\right) \in X^{2}$.

Remark 2.6 All of the coupled coincidence point results may be proved for a partially ordered cone $b$-metric space $(X, \sqsubseteq, d)$ by inserting well-known conditions like

(1) $F$ has the mixed $g$-monotone property and $g$ is $b$-continuous; and either

(a) $F$ is $b$-continuous, or

(b) $(X, d)$ is regular.

\section{Common coupled fixed point results}

The conditions of Theorem 2.1 are not enough to prove the existence of a common coupled fixed point for the mappings $F$ and $g$. By restricting to $w$-compatibility for $F$ and $g$, we obtain the following theorem.

Theorem 3.1 In addition to the hypotheses of Theorem 2.1, if $F$ and $g$ are w-compatible, then $F$ and $g$ have a unique common coupled fixed point. Moreover, a common coupled fixed point of $F$ and $g$ is of the form $(u, u)$ for some $u \in X$. 
Proof From Theorem 2.1, $F$ and $g$ have a coupled coincidence point $\left(x^{*}, y^{*}\right)$. Then $\left(g x^{*}, g y^{*}\right)$ is a coupled point of coincidence of $F$ and $g$ such that $g x^{*}=F\left(x^{*}, y^{*}\right)$ and $g y^{*}=F\left(y^{*}, x^{*}\right)$. First, we will show that the coupled point of coincidence is unique. Suppose that $F$ and $g$ have another coupled point of coincidence $\left(g x^{\prime}, g y^{\prime}\right)$ such that $g x^{\prime}=F\left(x^{\prime}, y^{\prime}\right)$ and $g y^{\prime}=$ $F\left(y^{\prime}, x^{\prime}\right)$, where $\left(x^{\prime}, y^{\prime}\right) \in X^{2}$. Then we have

$$
\begin{aligned}
d\left(g x^{*}, g x^{\prime}\right)= & d\left(F\left(x^{*}, y^{*}\right), F\left(x^{\prime}, y^{\prime}\right)\right) \\
\preceq & {\left[a_{1} d\left(g x^{*}, F\left(x^{*}, y^{*}\right)\right)+a_{2} d\left(g y^{*}, F\left(y^{*}, x^{*}\right)\right)\right] } \\
& +\left[a_{3} d\left(g x^{\prime}, F\left(x^{\prime}, y^{\prime}\right)\right)+a_{4} d\left(g y^{\prime}, F\left(y^{\prime}, x^{\prime}\right)\right)\right] \\
& +\left[a_{5} d\left(g x^{*}, F\left(x^{\prime}, y^{\prime}\right)\right)+a_{6} d\left(g y^{*}, F\left(y^{\prime}, x^{\prime}\right)\right)\right] \\
& +\left[a_{7} d\left(g x^{\prime}, F\left(x^{*}, y^{*}\right)\right)+a_{8} d\left(g y^{\prime}, F\left(y^{*}, x^{*}\right)\right)\right] \\
& +\left[a_{9} d\left(g x^{*}, g x^{\prime}\right)+a_{10} d\left(g y^{*}, g y^{\prime}\right)\right] \\
= & \left.a_{1} d\left(g x^{*}, g x^{*}\right)+a_{2} d\left(g y^{*}, g y^{*}\right)\right] \\
& +\left[a_{3} d\left(g x^{\prime}, g x^{\prime}\right)+a_{4} d\left(g y^{\prime}, g y^{\prime}\right)\right] \\
& +\left[a_{5} d\left(g x^{*}, g x^{\prime}\right)+a_{6} d\left(g y^{*}, g y^{\prime}\right)\right] \\
& +\left[a_{7} d\left(g x^{\prime}, g x^{*}\right)+a_{8} d\left(g y^{\prime}, g y^{*}\right)\right] \\
& +\left[a_{9} d\left(g x^{*}, g x^{\prime}\right)+a_{10} d\left(g y^{*}, g y^{\prime}\right)\right] \\
= & {\left[a_{5} d\left(g x^{*}, g x^{\prime}\right)+a_{6} d\left(g y^{*}, g y^{\prime}\right)\right] } \\
& +\left[a_{7} d\left(g x^{*}, g x^{\prime}\right)+a_{8} d\left(g y^{*}, g y^{\prime}\right)\right] \\
& +\left[a_{9} d\left(g x^{*}, g x^{\prime}\right)+a_{10} d\left(g y^{*}, g y^{\prime}\right)\right] . \\
&
\end{aligned}
$$

Hence,

$$
d\left(g x^{*}, g x^{\prime}\right) \preceq\left(a_{5}+a_{7}+a_{9}\right) d\left(g x^{*}, g x^{\prime}\right)+\left(a_{6}+a_{8}+a_{10}\right) d\left(g y^{*}, g y^{\prime}\right) .
$$

By a similar way, we can show that

$$
d\left(g y^{*}, g y^{\prime}\right) \preceq\left(a_{5}+a_{7}+a_{9}\right) d\left(g y^{*}, g y^{\prime}\right)+\left(a_{6}+a_{8}+a_{10}\right) d\left(g x^{*}, g x^{\prime}\right) .
$$

By adding inequalities (3.1) and (3.2), we get

$$
d\left(g x^{*}, g x^{\prime}\right)+d\left(g y^{*}, g y^{\prime}\right) \preceq\left(a_{5}+a_{6}+a_{7}+a_{8}+a_{9}+a_{10}\right)\left(d\left(g x^{*}, g x^{\prime}\right)+d\left(g y^{*}, g y^{\prime}\right)\right) .
$$

Since $\left(a_{5}+a_{6}+a_{7}+a_{8}+a_{9}+a_{10}\right)<1$, Lemma 1.5(1) shows that $d\left(g x^{*}, g x^{\prime}\right)+d\left(g y^{*}, g y^{\prime}\right)=\theta$. But $d\left(g x^{*}, g x^{\prime}\right) \succeq \theta$ and $d\left(g y^{*}, g y^{\prime}\right) \succeq \theta$. Hence, $d\left(g x^{*}, g x^{\prime}\right)=\theta$ and $d\left(g y^{*}, g y^{\prime}\right)=\theta$, that is,

$$
g x^{*}=g x^{\prime} \text { and } g y^{*}=g y^{\prime} \text {, }
$$

which implies the uniqueness of the coupled point of coincidence of $F$ and $g$, that is, $\left(g x^{*}, g y^{*}\right)$. By a similar way, we can prove that

$$
g x^{*}=g y^{\prime} \text { and } g y^{*}=g x^{\prime} .
$$


In view of (3.3) and (3.4), one can assert that

$$
g x^{*}=g y^{*}
$$

That is, the unique coupled point of coincidence of $F$ and $g$ is $\left(g x^{*}, g x^{*}\right)$.

Now, let $u=g x^{*}=F\left(x^{*}, y^{*}\right)$. Since $F$ and $g$ are $w$-compatible, then we have

$$
g u=g\left(g x^{*}\right)=g F\left(x^{*}, y^{*}\right)=F\left(g x^{*}, g y^{*}\right)=F\left(g x^{*}, g x^{*}\right)=F(u, u) .
$$

Then $(g u, g u)$ is a coupled point of coincidence, and also we have $(u, u)$ is a coupled point of coincidence. The uniqueness of the coupled point of coincidence implies that $g u=u$. Therefore $u=g u=F(u, u)$. Hence, $(u, u)$ is the unique common coupled fixed point of $F$ and $g$. This completes the proof.

Now, we present one example to illustrate our results.

Example 3.2 Let $X=[0,1]$ and $E=C_{\mathbb{R}}^{1}[0,1]$ with $\|u\|=\|u\|_{\infty}+\left\|u^{\prime}\right\|_{\infty}, u \in E$ and let $P=$ $\{u \in E: u(t) \geq 0$ on $[0,1]\}$. It is well known that this cone is solid but it is not normal. Define a cone $b$-metric $d: X \times X \rightarrow E$ by $d(x, y)(t)=|x-y|^{2} e^{t}$. Then $(X, d)$ is a complete cone $b$-metric space with the coefficient $s=2$. Let us define $F: X \times X \rightarrow X$ and $g: X \rightarrow X$ as $F(x, y)=\frac{1}{9} x+\frac{1}{7} y$ and $g(x)=\frac{1}{2} x$ for all $x \in X$. Now we obtain that

$$
\begin{aligned}
d(F(x, y), F(u, v))(t) & =\left|\frac{1}{9} x+\frac{1}{7} y-\frac{1}{9} u-\frac{1}{7} v\right|^{2} e^{t} \\
& =\left|\left(\frac{1}{9} x-\frac{1}{9} u\right)+\left(\frac{1}{7} y-\frac{1}{7} v\right)\right|^{2} e^{t} \\
& =\left|\frac{2}{9}\left(\frac{1}{2} x-\frac{1}{2} u\right)+\frac{2}{7}\left(\frac{1}{2} y-\frac{1}{2} v\right)\right|^{2} e^{t} \\
& \preceq 2\left(\left|\frac{2}{9}\left(\frac{1}{2} x-\frac{1}{2} u\right)\right|^{2} e^{t}+\left|\frac{2}{7}\left(\frac{1}{2} y-\frac{1}{2} v\right)\right|^{2} e^{t}\right) \\
& =\frac{8}{81}\left|\frac{1}{2} x-\frac{1}{2} u\right|^{2} e^{t}+\frac{8}{49}\left|\frac{1}{2} y-\frac{1}{2} v\right|^{2} e^{t} \\
& =\frac{8}{81} d(g x, g u)(t)+\frac{8}{49} d(g y, g v)(t),
\end{aligned}
$$

where $a_{9}=\frac{8}{81}, a_{10}=\frac{8}{49}, a_{i}=0, i=1,2, \ldots, 8$. Note that $2 s\left(a_{9}+a_{10}\right)=4\left(\frac{8}{81}+\frac{8}{49}\right)<2$, $F(X \times X) \subseteq g(X)$ and $g(X)$ is a complete subspace of $X$. Hence, the conditions of Theorem 2.1 are satisfied, that is, $F$ and $g$ have a coupled coincidence point $(0,0)$. Also, $F$ and $g$ are $w$-compatible at $(0,0)$. Hence, Theorem 3.1 shows that $(0,0)$ is the unique common coupled fixed point of $F$ and $g$.

Finally, we have the following result (immediate consequence of Theorems 2.1 and 3.1).

Theorem 3.3 Let $(X, d)$ be a complete cone $b$-metric space with the coefficient $s \geq 1$ relative to a solid cone P. Let $F: X^{2} \rightarrow X$ be a mapping and suppose that there exist nonnegative constants $a_{i} \in[0,1), i=1,2, \ldots, 10$, with $(s+1)\left(a_{1}+a_{2}+a_{3}+a_{4}\right)+s(s+1)\left(a_{5}+a_{6}+a_{7}+\right.$ 
$\left.a_{8}\right)+2 s\left(a_{9}+a_{10}\right)<2$ and $\sum_{i=1}^{10} a_{i}<1$ such that the following contractive condition holds for all $x, y, u, v \in X$ :

$$
\begin{aligned}
d(F(x, y), F(u, v)) \preceq & {\left[a_{1} d(x, F(x, y))+a_{2} d(y, F(y, x))\right] } \\
& +\left[a_{3} d(u, F(u, v))+a_{4} d(v, F(v, u))\right] \\
& +\left[a_{5} d(x, F(u, v))+a_{6} d(y, F(v, u))\right] \\
& +\left[a_{7} d(u, F(x, y))+a_{8} d(v, F(y, x))\right] \\
& +\left[a_{9} d(x, u)+a_{10} d(y, v)\right] .
\end{aligned}
$$

Then $F$ has a coupled fixed point $\left(x^{*}, y^{*}\right) \in X^{2}$. Moreover, the coupled fixed point is unique and of the form $\left(x^{*}, x^{*}\right)$ for some $x^{*} \in X$.

As consequences of Theorem 3.3, we have the following results which are the extension of main results of Sabetghadam et al. [34] to cone $b$-metric spaces.

Corollary 3.4 Let $(X, d)$ be a complete cone b-metric space with the coefficient $s \geq 1$ relative to a solid cone P. Let $F: X^{2} \rightarrow X$ be a mapping and suppose that there exist nonnegative constants $k, l$ with $k+l<\frac{1}{s}$ such that the following contractive condition holds for all $x, y, u, v \in X:$

$$
d(F(x, y), F(u, v)) \preceq k d(x, u)+l d(y, v) .
$$

Then $F$ has a coupled fixed point $\left(x^{*}, y^{*}\right) \in X^{2}$. Moreover, the coupled fixed point is unique and of the form $\left(x^{*}, x^{*}\right)$ for some $x^{*} \in X$.

Corollary 3.5 Let $(X, d)$ be a complete cone b-metric space with the coefficient $s \geq 1$ relative to a solid cone P. Let $F: X^{2} \rightarrow X$ be a mapping and suppose that there exist nonnegative constants $k, l$ with $k+l<\frac{2}{s+1}$ such that the following contractive condition holds for all $x, y, u, v \in X:$

$$
d(F(x, y), F(u, v)) \preceq k d(x, F(x, y))+l d(u, F(u, v)) .
$$

Then $F$ has a coupled fixed point $\left(x^{*}, y^{*}\right) \in X^{2}$. Moreover, the coupled fixed point is unique and of the form $\left(x^{*}, x^{*}\right)$ for some $x^{*} \in X$.

Corollary 3.6 Let $(X, d)$ be a complete cone b-metric space with the coefficient $s \geq 1$ relative to a solid cone $P$. Let $F: X^{2} \rightarrow X$ be a mapping and suppose that there exist nonnegative constants $k, l$ with $k+l<\frac{2}{s(s+1)}$ such that the following contractive condition holds for all $x, y, u, v \in X$ :

$$
d(F(x, y), F(u, v)) \preceq k d(x, F(u, v))+l d(u, F(x, y)) .
$$

Then $F$ has a coupled fixed point $\left(x^{*}, y^{*}\right) \in X^{2}$. Moreover, the coupled fixed point is unique and of the form $\left(x^{*}, x^{*}\right)$ for some $x^{*} \in X$. 


\section{Competing interests}

The authors declare that they have no competing interests.

\section{Authors' contributions}

All authors contributed equally and significantly in writing this paper. All authors read and approved the final manuscript.

\section{Acknowledgements}

The authors would like to acknowledge the financial support received from Faculty of Science and Technology, Universiti Kebangsaan Malaysia. The authors thank the referee for his/her careful reading of the manuscript and useful suggestions.

\section{Received: 6 March 2013 Accepted: 19 June 2013 Published: 5 July 2013}

\section{References}

1. Bakhtin, IA: The contraction mapping principle in almost metric spaces. In: Functional Analysis, vol. 30, pp. 26-37. Gos. Ped. Inst. Ulyanowsk, Ulyanowsk (1989)

2. Khamsi, MA, Hussain, N: KKM mappings in metric type spaces. Nonlinear Anal. 73, 3123-3129 (2010)

3. Czerwik, S: Nonlinear set-valued contraction mappings in b-metric spaces. Atti Semin. Mat. Fis. Univ. Modena 46, 263-276 (1998)

4. Boriceanu, M, Bota, M, Petrusel, A: Mutivalued fractals in b-metric spaces. Cent. Eur. J. Math. 8(2), 367-377 (2010)

5. Bota, M, Molnar, A, Csaba, V: On Ekeland's variational principle in b-metric spaces. Fixed Point Theory 12, 21-28 (2011)

6. Aydi, H, Bota, M, Karapinar, E, Mitrović, S: A fixed point theorem for set-valued quasicontractions in $b$-metric spaces. Fixed Point Theory Appl. 2012, 88 (2012)

7. Jovanović, M, Kadelburg, Z, Radenović, S: Common fixed point results in metric-type spaces. Fixed Point Theory Appl. 2010, Article ID 978121 (2010)

8. Kantorovich, LV: The method of successive approximations for functional equations. Acta Math. 71, 63-77 (1939)

9. Kantorovich, LV: The majorant principle and Newton's method. Dokl. Akad. Nauk SSSR 76, 17-20 (1951)

10. Kantorovich, LV: On some further applications of the Newton approximation method. Vestn. Leningr. Univ., Mat. Mekh. Astron. 12(7), 68-103 (1957)

11. Vandergraft, J: Newton method for convex operators in partially ordered spaces. SIAM J. Numer. Anal. 4, 406-432 (1967)

12. Zabreiko, PP: K-Metric and K-normed spaces: survey. Collect. Math. 48, 852-859 (1997)

13. Deimling, K: Nonlinear Functional Analysis. Springer, Berlin (1985)

14. Mohebi, H: Topical functions and their properties in a class of ordered Banach spaces. In: Continuous Optimization: Current Trends and Modern Applications, pp. 343-361. Springer, New York (2005). Part II of the book

15. Huang, LG, Zhang, X: Cone metric spaces and fixed point theorems of contractive mappings. J. Math. Anal. Appl. 332(2), 1468-1476 (2007)

16. Abbas, M, Jungck, G: Common fixed point results for noncommuting mappings without continuity in cone metric spaces. J. Math. Anal. Appl. 341, 416-420 (2008)

17. Abbas, M, Rhoades, BE: Fixed and periodic point results in cone metric spaces. Appl. Math. Lett. 22(4), $511-515$ (2009)

18. Abbas, M, Jovanović, M, Radenović, S, Sretenović, A, Simić, S: Abstract metric spaces and approximating fixed points of a pair of contractive type mappings. J. Comput. Anal. Appl. 13(2), 243-253 (2011)

19. Aranđelović, ID, Kečkić, DJ: A counterexample on a theorem of Khojasteh, Goodarzi and Razani. Fixed Point Theory Appl. 2010, Article ID 470141 (2010). doi:10.1155/2010/470141

20. Beg, I, Azam, A, Arshad, M: Common fixed point for maps on topological vector space valued cone metric spaces. Int J. Math. Math. Sci. 2009, Article ID 560264 (2009). doi:10.1155/2009/560264

21. Hussain, N, Shah, MH, Radenović, S: Fixed points of weakly contractions through occasionally weak compatibility. J. Comput. Anal. Appl. 13(3), 532-543 (2011)

22. Ilić, D, Rakočević, V: Common fixed point for maps on cone metric space. J. Math. Anal. Appl. 341, 876-882 (2008)

23. Janković, S, Kadelburg, Z, Radenović, S, Rhoades, BE: Assad-fixed-type point theorems for a pair of nonself mappings on cone metric spaces. Fixed Point Theory Appl. 2009, Article ID 761086 (2009). doi:10.1155/2009/761086

24. Jungck, G, Radenović, S, Radojević, S, Rakočević, V: Common fixed point theorems for weakly compatible pairs on cone metric spaces. Fixed Point Theory Appl. 2009, Article ID 643840 (2009)

25. Abbas, M, Khan, MA, Radenović, S: Common coupled fixed point theorems in cone metric spaces for $w$-compatible mappings. Appl. Math. Comput. 217, 195-202 (2010)

26. Hussain, N, Shah, MH: KKM mappings in cone b-metric spaces. Comput. Math. Appl. 62(4), 1677-1684 (2011)

27. Cvetković, AS, Stanić, MP, Dimitrijević, S, Simić, S: Common fixed point theorems for four mappings on cone metric type space. Fixed Point Theory Appl. 2011, Article ID 589725 (2011)

28. Stanić, MP, Cvetković, AS, Simić, S, Dimitrijević, S: Common fixed point under contractive condition of Ćirić's type on cone metric type spaces. Fixed Point Theory Appl. 2012, 35 (2012)

29. Shah, MH, Simić, S, Hussain, N, Sretenović, A, Radenović, S: Common fixed points for occasionally weakly compatible pairs on cone metric type spaces. J. Comput. Anal. Appl. 14(2), 290-297 (2012)

30. Huang, $\mathrm{H}, \mathrm{Xu}, \mathrm{S}$ : Fixed point theorems of contractive mappings in cone $b$-metric spaces and applications. Fixed Point Theory Appl. 2012, 220 (2012)

31. Hussain, N, Đorić, D, Kadelburg, Z, Radenović, S: Suzuki-type fixed point results in metric type spaces. Fixed Point Theory Appl. 2012, 126 (2012)

32. Bhaskar, TG, Lakshmikantham, V: Fixed point theorems in partially ordered metric spaces and applications. Nonlinear Anal., Theory Methods Appl. 65(7), 1379-1393 (2006)

33. Lakshmikantham, V, Ćirić, L: Coupled fixed point theorems for nonlinear contractions in partially ordered metric spaces. Nonlinear Anal., Theory Methods Appl. 70(12), 4341-4349 (2009)

34. Sabetghadam, F, Masiha, HP, Sanatpour, AH: Some coupled fixed point theorems in cone metric spaces. Fixed Point Theory Appl. 2009, Article ID 125426 (2009) 
doi:10.1186/1687-1812-2013-177

Cite this article as: Fadail and Ahmad: Coupled coincidence point and common coupled fixed point results in cone b-metric spaces. Fixed Point Theory and Applications 2013 2013:177.

Submit your manuscript to a SpringerOpen ${ }^{\circ}$ journal and benefit from:

- Convenient online submission

- Rigorous peer review

- Immediate publication on acceptance

Open access: articles freely available online

- High visibility within the field

- Retaining the copyright to your article

Submit your next manuscript at $\gg$ springeropen.com 